Canadian University Music Review

Revue de musique des universités canadiennes

\title{
Carlo Caballero. Fauré and French Musical Aesthetics. Cambridge, NY: Cambridge University Press, 2001. xi, 333 p. ISBN 0-521-78107-8 (couverture cartonnée)
}

\section{Christopher Moore}

Volume 23, numéro 1-2, 2003

URI : https://id.erudit.org/iderudit/1014532ar

DOI : https://doi.org/10.7202/1014532ar

Aller au sommaire du numéro

\section{Éditeur(s)}

Canadian University Music Society / Société de musique des universités canadiennes

\section{ISSN}

0710-0353 (imprimé)

2291-2436 (numérique)

Découvrir la revue

Citer ce compte rendu

Moore, C. (2003). Compte rendu de [Carlo Caballero. Fauré and French Musical Aesthetics. Cambridge, NY: Cambridge University Press, 2001. xi, 333 p. ISBN 0-521-78107-8 (couverture cartonnée)]. Canadian University Music Review / Revue de musique des universités canadiennes, 23(1-2), 248-253. https://doi.org/10.7202/1014532ar

All Rights Reserved (C Canadian University Music Society / Société de musique des universités canadiennes, 2004
Ce document est protégé par la loi sur le droit d'auteur. L'utilisation des services d'Érudit (y compris la reproduction) est assujettie à sa politique d'utilisation que vous pouvez consulter en ligne.

https://apropos.erudit.org/fr/usagers/politique-dutilisation/ 
L'ouvrage est augmenté d'une chronologie, d'une bibliographie et de photos. Il constitue l'un des premiers témoignages autobiographiques d'un chansonnier au Québec, en dépit du fait que la contribution autobiographique de sa carrière de chansonnier reste limitée. Bruno a fait un travail de longue haleine en dépouillant une documentation exhaustive sans chercher à louanger une figure mythique. Même s'il ne contribue pas de manière particulière à l'étude des textes de Dor dans son ensemble, l'ouvrage autobiographique offre des pistes aux chercheurs qui souhaiteraient aborder l'une ou l'autre des œuvres de l'écrivain en plus de synthétiser la pensée intime d'un chansonnier assez négligé de notre culture québécoise, que la publication des essais (entre 1996 et 2001) avait su relancer.

Jean-Nicolas DeSurmont

Carlo Caballero. Fauré and French Musical Aesthetics. Cambridge, NY: Cambridge University Press, 2001. xi, 333 p. ISBN 0-521-78107-8 (couverture cartonnée).

Carlo Caballero est professeur associé à la Faculté de Musique de l'Université du Colorado. Fauré and French Musical Aesthetics, basé sur sa thèse de doctorat soutenue à l'Université de Pennsylvanie en 1996, fait partie de la collection « Music in the Twentieth Century 》 éditée par Arnold Whitall. Carlo Caballero est très actif dans la recherche sur la musique de la fin du XIXe siècle en France, et a rédigé des chapitres pour deux livres : Regarding Fauré et The Arts Entwined: Music and Painting in the Nineteenth Century 1.

Pour le lecteur au fait de la littérature fauréenne, il est impossible de ne pas associer deux mots du titre du premier livre de Carlo Caballero («Fauré » et " esthétique ") aux écrits du philosophe Vladimir Jankélévitch, qui eurent un impact si important sur la recherche sur ce compositeur et sur la musicologie française en général ${ }^{2}$. Avec un langage à la fois savant et poétique, Jankélévitch a communiqué aux lecteurs son amour pour cette musique tout en leur offrant un panorama très particulier de l'esthétique fauréenne. Sa vision de cette esthétique se révèle par l'exploration de liens intertextuels et stylistiques puisés dans l'ensemble de l'œuvre du compositeur. Ses ouvrages se lisent comme des poèmes métaphysiques dans lesquels l'intuition sensible d'un musicien averti semble très souvent être à la base de conclusions sinon toutes convaincantes, du moins toujours stimulantes. À l'Américain Carlo Caballero ne font défaut ni l'amour pour cette musique ni la sensibilité requise pour écrire une telle monographie. Même si ses conclusions n'apportent pas de grandes modifications à ce que l'on peut lire dans Jankélévitch ou Jean-Michel Nectoux ${ }^{3}$, la

1 Carlo Caballero, "Fauré's religion and La Chanson d'Ève ", dans Regarding Fauré, sous la dir. de Tom Gordon (Amsterdam : Gordon \& Breach, 1999); «In the Toils of Queen Omphale : Saint-Saens' Painterly Refiguration of the Symphonic Poem ", dans The Arts Entwined : Music and Painting in the Nineteenth Century, sous la dir. de Marsha L. Morton et Peter L. Schmunk (New York : Garland, 2000).

2Vladimir Jankélévitch, Fauré et l'inexprimable (Paris : Plon, 1974); La musique et l'ineffable (Paris : A. Colin, 1961).

3 Jean-Michel Nectoux, Gabriel Fauré : les voix du clair-obscur (Paris : Flammarion, 1990). 
méthode qu'il emploie pour examiner son sujet aide à renouveler et à approfondir la réflexion sur l'esthétique fauréenne et sur le milieu culturel (musical, littéraire, philosophique et journalistique) qui l'a influencé. Les quatre premiers chapitres sont liés par le thème de la " vocation artistique " et traitent de : 1) la sincérité; 2) l'innovation et la tradition; 3) l'originalité, l'influence et le renouveau de soi; 4) l'homogénéité. Le cinquième chapitre explore le développement religieux et spirituel du compositeur, tandis que le chapitre final réinterprète un thème souvent évoqué dans les discussions sur Fauré la qualité évasive et insaisissable de sa musique.

L'approche de Caballero est typique des recherches actuelles en Amérique du Nord sur l'histoire de la musique en France durant la $\mathrm{III}^{\mathrm{e}}$ République. L'auteur propose une esthétique du compositeur par le biais d'une « contextualisation " riche en documentation sur l'époque - correspondances, études, mémoires - , souvent mise en parallèle avec d'autres sources - journalistiques ou archivistiques - plus difficilement accessibles. Pour Caballero, le recours à cette documentation est rendue nécessaire par le silence presque obstiné entretenu par Fauré en ce qui concerne sa propre musique et, par extension, son esthétique musicale. Par conséquent, cette méthodologie donne lieu à une étude très détaillée qui place la personnalité de Fauré au centre d'une vaste interrogation sur l'esthétique musicale de son époque. Même s'il est vrai que Caballero se perd par moments dans la densité de cette méthode, la rhétorique qu'il emploie et l'intelligence dont il fait preuve pour toujours éclairer ses observations à la lumière de la pensée fauréenne sont impressionnantes.

Malgré les fréquentes récurrences du mot " sincère » dans les correspondances et textes relatifs à la musique de la fin du siècle en France, personne, jusqu'à maintenant, n'a tenté d'élucider ce que signifiait le concept de sincérité en termes d'esthétique musicale pour les compositeurs de cette époque. Dans le premier chapitre de son livre, Caballero explore la notion de sincérité et la possibilité de sa traduction dans des auvres musicales de la fin du siècle, en s'appuyant sur des témoignages d'élèves de Fauré, afin de démontrer que la sincérité était un élément clé de la pensée musicale fauréenne. L'auteur examine la signification de la sincérité au tournant du siècle par une analyse des principaux textes philosophiques traitant du sujet. Il montre que, dans ce milieu, la musique était de facto assimilée à la traduction de " la vie intérieure » (p. 40) ${ }^{4}$. Caballero prend l'exemple de Henri Bergson (1859-1941) et illustre comment ce philosophe a utilisé l'analogie musicale dans ses écrits pour clarifier sa conception de " la vie intérieure » et de " la durée pure » (p. 38-41). Il explique que, pour Bergson, la musique était « une révélation adéquate de cette même vie intérieure qu'il désirait atteindre, en tant que philosophe, par les moyens de la pensée spéculative » (p. 40). Cette assimilation quasi complète de la musique avec la « vie en profondeur » ouvre un champ important à la notion de sincérité; car, si le rôle de la musique est de traduire l'intériorité et l'expérience subjective, la sincérité d'expression devient alors la clé de voûte de leur juste représentation. Dans un tel climat philosophique, explique Caballero, il était tout à fait naturel que Fauré voue un véritable culte à la sincérité dans la composition musicale.

4Sauf indication contraire, les citations sont traduites par l'auteur de cette recension. 
La sincérité ayant aussi pris une place importante dans le message pédagogique de Fauré, Caballero examine l'influence marquante qu'elle produisit sur des élèves tels Nadia Boulanger, Charles Kœchlin et André Messager. Surtout, il démontre pourquoi la musique de Fauré a été reconnue pour sa sincérité par des compositeurs et des critiques à la fin du siècle. Trois raisons sont à la base de cette appréciation : le mépris de Fauré pour les vogues musicales, son dédain à l'égard du mercantilisme dans l'art, et enfin son refus de jouer à l'arriviste en offrant des innovations au public à seule fin de faire du tape-à-l'œil. Cette persévérance dans une vision personnelle et non corrompue de l'art comporte des conséquences stylistiques, et Caballero examine dans son quatrième chapitre l'une des plus importantes de ces conséquences, l'homogénéité, et sa place dans le monde artistique à la fin du XIX ${ }^{e}$ siècle.

Caballero explique que, pour un musicien travaillant dans un milieu musical comptant beaucoup sur la sincérité dans la composition, la perception par la critique « d'une fragmentation ou d'un manque d'intégrité dans l'expression de soi avait des conséquences désastreuses » (p. 137). Claude Debussy en a subi les conséquences, nous rappelle Caballero, après les premières d'Ibéria et de Rondes de printemps en 1910, alors que certains critiques se trouvèrent déconcertés par ce qu'ils percevaient comme un manque de continuité stylistique reliant ces auvres à celles de la période de Pelléas (p. 139-41). La musique de Fauré n'a pas provoqué de pareilles objections, car, comme l'indique Caballero, les critiques semblent y avoir reconnu « une courbe de développement compréhensible » dans l'évolution de son style (p. 148). Tout de même, l'homogénéité n'était pas suffisante à elle seule pour convaincre les critiques : Caballero souligne que le compositeur devait posséder une foi inébranlable en un idéal artistique, tout en étant capable de renouvellement et d'innovation dans son expression de soi. Caballero illustre comment cette tension entre l'homogénéité et l'innovation préoccupait les artistes de la fin du siècle par une exploration de quelques passages clés tirés de $\dot{A}$ la recherche $d u$ temps perdu. Caballero cite les observations de Marcel à l'écoute de la " sonate de Vinteuil ", quand le protagoniste discerne, sous les innovations et différences qui marquent l'art de Vinteuil, les profondes ressemblances, parfois involontaires, qui existent dans toute l'œuvre du compositeur fictif (p. 155). Les mêmes " ressemblances involontaires", explique Caballero, abondent dans l'œuvre musicale de Fauré, ce qui se vérifie non seulement avec des phrases musicales telles que les thèmes de "Lydia " ou d'" Odysse ", mais aussi dans l'emploi par Fauré de certains gestes musicaux récurrents. Comme l'auteur le souligne, Fauré était sensible au point d'être quelque peu angoissé par l'homogénéité de sa musique, et ses propres commentaires à ce sujet sont révélateurs : « Je voudrais bien que ma musique fût aussi diverse. Il me semble que je me répète constamment et que je ne trouve pas un accent sensiblement différent de ceux que j'ai déjà exprimés ${ }^{5}$. Il semble que le désir de rester homogène comme expression de sincérité et le désir tout aussi fort d'innover

5Gabriel Fauré, Lettres intimes (Paris : La Colombe, 1951), 87. 
sous les contraintes de cette homogénéité furent problématiques pour le compositeur; en effet, ces œuvres témoignent d'une fascinante synthèse de ces deux tendances opposées. On pourrait souhaiter que des études ultérieures viennent examiner plus en profondeur cette dialectique fauréenne.

Ayant montré la présence de certaines constantes esthétiques dans la totalité de l'œuvre du compositeur, Caballero se penche, dans le cinquième chapitre, sur une œuvre en particulier, La chanson d'Ève, et un thème, la religion, avec le but de comprendre " comment l'art de Fauré nous offre des preuves de ses croyances religieuses » (p. 170). Ce cycle de mélodies, écrit entre 1906 et 1910, met en musique les vers de Charles Van Lerberghe (1861-1907), poète belge symboliste. Anticlérical farouche, Van Lerberghe avoua qu'il ne put jamais lire l'histoire de Dieu dans la Bible sans horreur et dégoût. De ce fait, son interprétation de l'histoire d'Ève dans le jardin d'Eden introduit des changements notoires. Ėve apparaît seule dans le jardin (Van Lerberghe estimait que l'Homme n'était rien d'autre qu'un " accident clitorique ${ }^{6} "$ ), où elle connaît des gratifications à la fois physiques et intellectuelles. Nulle faute originelle n'est commise et nul châtiment n'est administré; Ève, ayant célébré le jour, s'éteint, pacifique, dans le calme du néant.

Caballero suggère que cette œuvre, écrite suivant la nomination de Fauré à la tête du Conservatoire, "marque le point tournant entre le catholicisme décroissant de Fauré et son athéisme naissant " (p. 198). Selon Caballero, $L a$ chanson d'Ève serait un exemple de panthéisme " poétique » (p. 198), une hypothèse à laquelle Nectoux a déjà fait allusion ${ }^{7}$. Pour soutenir son argument, Caballero explore « la portée et le développement des idées qui figuraient sous le nom de "panthéisme" en France au dix-neuvième siècle " (p. 195). Pour lui, la définition du terme par Paul Bourget (" la communion de l'âme et la nature $\left.{ }^{8} »\right)$, caractérise l'approche de Fauré à l'égard de ce cycle. Mais, bien que La chanson d'Ève soit fortement inspirée de cette " communion », le terme " panthéisme " me semble trop général pour véritablement caractériser la sensibilité fauréenne au moment de la composition du cycle. Il est difficile d'y voir un aveu explicite de panthéisme quand il existe tellement de moments nourris de la même sensibilité dans l'ensemble de l'œuvre du musicien. Par exemple, "C'est l'extase... ", de 1891, ou «L'inscription sur le sable » du Jardin clos, de 1915, célèbrent la communion de l'âme (amoureuse ou mourante) avec la nature. Il est aussi important de souligner que le panthéisme inhérent aux poèmes de Van Lerberghe n'était pas forcément l'élément ayant le plus séduit le compositeur. Nous savons que Fauré choisissait scrupuleusement ses textes et appréciait les poèmes autant pour leur atmosphère que pour la musicalité de leurs vers. N'est-il pas possible que Fauré eut été simplement

6Lettre de Charles Van Lerberghe à Albert Mockel (6 août 1902). Il continue : "Ève était bissexuée [sic], hermaphrodite comme un lis. Elle s'est fécondée elle-même, évidemment ". Charles Van Lerberghe, Lettres à Albert Mockel (1887-1906) (Bruxelles : Editions Labor, 1986), 298-99.

7 Jean-Michel Nectoux, Fauré, 182.

8Paul Bourget, Nouveaux essais de psychologie contemporaine (Paris : Lemerre, 1886), 120. 
attiré par la poésie de Van Lerberghe pour « la fluidité, la légèreté aérienne de ses phrases et surtout l'allure timide, gauche, sans rien qui pèse et qui pose ", comme la définissait le poète lui-même ${ }^{9}$ ? $\mathrm{Ne}$ trouve t-on pas dans cette description toutes les qualités essentielles de l'art fauréen?

Caballero admet que ses arguments portant sur le développement spirituel du compositeur ne peuvent être concluants, et il est regrettable que ses thèses se heurtent parfois contre un mur de silence documentaire. De ce fait, malgré le grand effort de contextualisation, ce livre n'apporte que très peu de nouveaux renseignements sur l'esthétique du compositeur qui n'aient pas déjà été relevés par d'autres auteurs. Certes, le livre de Caballero présente les thèmes de son interrogation d'une façon systématique et détaillée qui nous change un peu de l'approche de Jankélévitch, mais les résultats de ses recherches demeurent souvent les mêmes que celles de ce dernier. Cependant, cela est dû sans doute plus à la qualité insaisissable de son sujet qu'à un quelconque manque de rigueur intellectuelle chez l'auteur. Car Gabriel Fauré et sa musique sont à l'image d'Ève ou de Mélisande : "Ils ont si peur de s'imposer! Ils furent toujours si timides et si discrets, si merveilleusement lunaires! Ils chuchotent, ils glissent, ils frôlent les nuages... ${ }^{10}$ ". Essayer de réduire cet art à des " conclusions » soi-disant scientifiques aurait le même effet que d'empaler un papillon : le vider de sa sève vitale.

Heureusement, Caballero est sensible au devenir musical de cette sève fauréenne. L'auteur dépasse les observations de ses collègues français dans sa capacité d'argumenter en faveur du compositeur en ce qui concerne l'originalité et l'importance de sa position artistique (jusqu'ici assez marginalisée, surtout en Amérique) à l'égard de notre conception moderne (ou encore, postmoderne) de l'évolution musicale en Europe au début du $\mathrm{XX}^{\mathrm{e}}$ siècle. Surtout maintenant, alors que l'on constate que la musique contemporaine englobe des tendances à la fois tonales et atonales, la musique de Fauré (qui, malgré les "progrès " introduits par Schoenberg et Stravinsky, n'a jamais complètement abandonné un style d'écriture formé par le XIX ${ }^{e}$ siècle) réapparaît non plus comme un anachronisme au milieu du modernisme émergeant, mais comme une source de potentialité pour maintenant et le futur (p. 8).

Ce livre, élégamment écrit dans un anglais à la fois recherché et exact, est une belle victoire pour la recherche fauréenne qui, malheureusement, suit toujours d'assez loin en quantité celles entreprises sur ses compatriotes Debussy ou Ravel. Peut-être est-ce l'homogénéité que l'on retrouve dans la musique de Fauré qui explique que beaucoup de ses œuvres restent complètement ignorées par la critique et les interprètes? Pour cette raison, $\mathrm{j}$ 'aurais souhaité que Caballero utilise davantage d'exemples musicaux (en 256 pages de texte, il nous en donne seulement 22) pour illustrer ses propos et partager ce répertoire injustement méconnu. Malgré cette réserve, $\mathrm{j}$ 'hésite pourtant à croire que cela eût vraiment aidé à une plus grande

9Lettre de Charles Van Lerberghe à Fernand Severin (23 juillet 1899). Charles Van Lerberghe, Lettres à Fernand Severin (Bruxelles : La Renaissance du Livre, 1924), 135.

10 Vladimir Jankélévitch, Fauré, 263. 
diffusion des œuvres du compositeur. La musique de Fauré demeure un art de l'équivoque, de l'impalpable et de l'évasion; une musique peu faite pour plaire à la majorité. N'est-ce pas cette pudeur, ce refus de s'exprimer haut et fort, qui la rend, pour certains, encore plus délectable?

Christopher Moore 